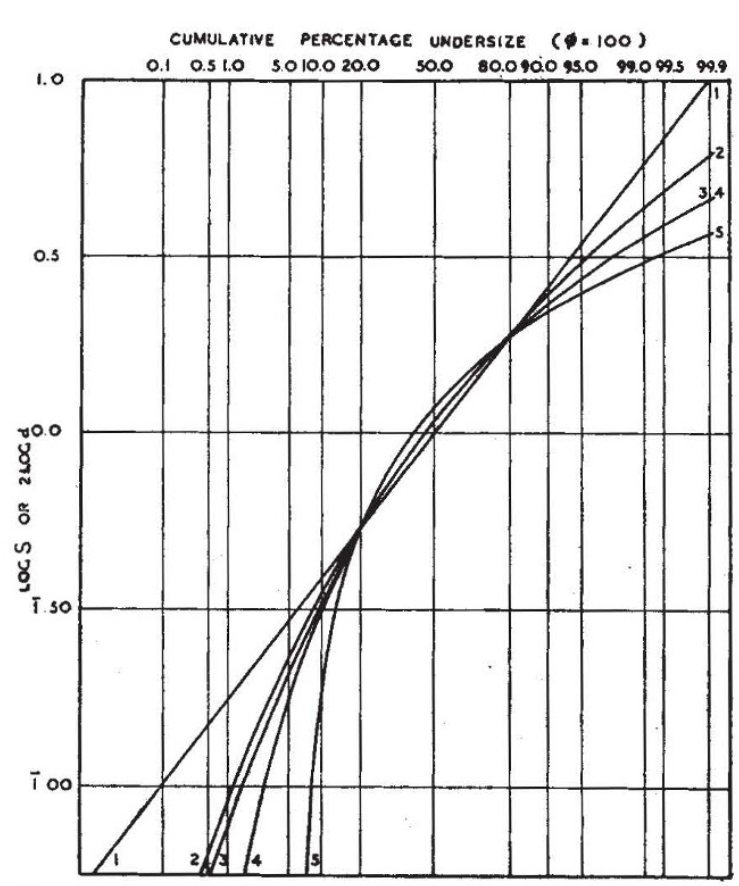

CURVE 1. LOGARITHMIC PROBABIHITY (LOGNORMAL DISTRIBUTION). CURVe 2. EQUATION (1), $n=1$.

CURVE 3. ROSIN-RAMMLER RELATION IN $S$ or $d$ :

$$
\varphi=1-\exp \left(-\left(\frac{S}{a}\right)^{b}\right) \text {. }
$$

CURVE 4. ARITHMETIC PROBABIhITY (NORMaL OR GadSSIAN DISTRIBUTION) IN $d$.
CURVE 5. ARITHMETIC PROBABILITY (NORMAL OR GAUSSIAN DISTRIBCTION) IN $S$.

about one half as great for $n=4$ as for $n=1$ ) and the Rosin-Rammle. function, and it may be interesting to quote from the paper by C. Er Lapple ${ }^{1}$, from which this figure is partly taken : "Usually distributions obtained in practice will yield curves that are intermediate between

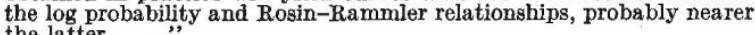

The general integrated form of equation (1) is not obtainable except for integral values of $n$, but the particular integral $\int_{0}^{\infty} Y_{S} \cdot d S$ and many derivatives of this which appear in sedimentation and dust-transport problems arefeasily evaluated.

An important limitation of the function is that there is a relatively low upper limit to the degree of dispersion, roughly equivalent to a standard deviation of 0.4 on the closest logarithmic probability distribution. The range is, however, wide enough to cover a considerable number of cases, especially where the material remains in suspension for any considerable time.

Industrial Health Research Board,

O. M. LIDWELI

c/o London School of Hygiene and Tropical Medicine,

$$
\text { Keppel Street, London, W.C.1. }
$$

${ }^{1}$ Lapple, C. E., Heating, Piping and Air Conditioning, 18, 113 (1946).

\section{The Lorentz Transformation}

IN a letter entitled “A Simple Proof of the Lorentz 'Transformation" appearing in Nature ${ }^{1}$, an error has been introduced into the matrix algebra, the correction of which requires a certain recasting of the proof.

From postulate $(P 1)$, it follows that

$$
\begin{aligned}
& f_{11}(-v)=\frac{f_{22}(v)}{D(v)}, f_{12}(-v)=-\frac{f_{12}(v)}{D(v)}, \\
& \quad f_{21}(-v)=-\frac{f_{21}(v)}{D(v)}, f_{22}(-v)=\frac{f_{11}(v)}{D(v)}(1,2,3,4)
\end{aligned}
$$

By writing $-v$ for $v$ in (2), (3), or (1) and (4), it may be seen that $D(v) D(-v)=1$. On the other hand, the author's derivation of the minant $D(-v)$, cannot be accepted since

$$
D(-v)=\frac{1}{(D(v))^{2}}\left|\begin{array}{cc}
f_{22}(v) & -f_{12}(v) \\
-f_{21}(v) & f_{11}(v)
\end{array}\right|=\frac{1}{D(v)},
$$

which has already been proved.

The proof of the Lorentz transformation may be continued as follows :
From postulate $(P 2)$, it follows that $\frac{f_{12}(v)}{f_{21}(v)}$ and $\frac{f_{11}(v)-f_{22}(v)}{f_{12}(v)}$ are invariant and we write $\frac{f_{12}(v)}{f_{21}(v)}=C^{2}$. By definition $v=-\frac{f_{12}(v)}{f_{11}(v)}$.

$$
\begin{gathered}
\therefore \quad D(v)=f_{11}(v)\left(f_{22}(v)-\frac{v^{2}}{c^{2}} f_{11}(v)\right) \\
\text { and } D(-v)=f_{11}(-v)\left(f_{22}(-v)-\frac{v^{2}}{c^{2}} f_{11}(-v)\right) \\
=\frac{f_{22}(v)}{(D(v))^{2}}\left(f_{11}(v)-\frac{v^{2}}{c^{2}} f_{22}(v)\right) .
\end{gathered}
$$

From this it follows that $f_{92}(v)= \pm f_{11}(v)$.

Now if $f_{22}(v)=-f_{11}(v), \frac{f_{11}(v)-f_{22}(v)}{f_{12}(v)}=-\frac{2}{v}$, which contradicts (P2).

$$
\begin{aligned}
\therefore f_{22}(v)= & f_{11}(v), \text { and further, } f_{11}(v) f_{11}(-v)=\frac{1}{1-v^{2} / c^{2}} . \\
& \therefore F(v)=f_{11}(v)\left[\begin{array}{cc}
1 & -v \\
-v / c^{2} & 1
\end{array}\right], \cdot \cdot(3) \\
& \text { and } f_{11}(v) f_{11}(-v)=\frac{1}{1-v^{2} / c^{2}}, \cdot \cdot\left(3 a_{c}\right)
\end{aligned}
$$$$
\text { (thus } f_{11}(v)=\frac{\lambda v}{\sqrt{1-v^{2} / c^{2}}} \text { satisfles }\left(3 a_{c}\right) \text { ). }
$$

To complete the proof it is evidently necessary to introduce a further postulate, and it would therefore appear that the conclusion drawn in the original letter, namely, that the Lorentz transformation has a more general signiflcance than is widely believed, requires further examination.

Post Office Engineering Research Station,

$$
\begin{gathered}
\text { Dollis Hill, } \\
\text { London, N.W.2. } \\
\text { June 6. }
\end{gathered}
$$

P. J. HILTON

${ }^{1}$ Strauss, M. D. H., Nature, 157, 516 (1946). The derivation of the Lorentz transformation, as given in my
previous communication, is marred by a mistake, pointed out by Mrevious communication, H. Hilton, which Is marred

The two postulates for the transformation matrix, namely,

$$
\begin{gathered}
F(-v)=F(v)^{-1}, \ldots . \\
F(v) F^{\prime}\left(v^{\prime}\right)=F\left(v^{\prime}\right) F(v), .
\end{gathered}
$$

together with the deflnition of $v$, lead to

$$
F(v)=f_{11}(v)\left(\begin{array}{cc}
1 & -v \\
-v / C^{2} & 1
\end{array}\right), .
$$

with

$$
f_{11}(v) f_{11}(-v)=\left[1-v^{2} / C^{2}\right]^{-1} . \quad .
$$

If we now assume the space to be isotropic, the equations

$$
\begin{aligned}
& x^{\prime}=f_{11}(v) x+f_{12}(v) t, . . \\
& t^{\prime}=f_{21}(v) x+f_{22}(v) t, . . .
\end{aligned}
$$

must be invariant under the substitution

$$
x \rightarrow-x, \quad x^{\prime} \rightarrow-x^{\prime}, \quad v \rightarrow-v,
$$

so that

$$
f_{11}(v)=f_{11}(-v), . . .
$$

which together with $\left(3 a_{c}\right)$ gives the desired result.

It may be worth noting that $(P 3)$ is not required to establish the group property of the transformations $F(v)$ and the formula for the composition of veloeities ; both follow from (3) and the definition of $v$. It would therefore appear that the conclusion drawn in my previous letter is essentially correct. I should also like to point out that the traditional method of using, in addition to the postulate of relativity, the postulate of the constancy of the velocity of light, was perfectiy rational at a time when the two postulates were thought to contradict each other. Yet when one learns afterwards that the Galilean transformation follows from the Lorentz transformation by the specification $C=\infty$, one naturally wonders why it should be necessary to invoke a postulate which leaves no trace in the result. The answer, as we have seen, is that no such postulate is required.

In the meantime, I have learned to my satisfaction that other authors have come to the same conclusion. Pars ${ }^{1}$ has derived the Lorentz transformation from the postulates that the space is isotropic and that the transformations $F(v)$ form a group. Frank and Rothe ${ }^{2}$ have treated the problem in a somewhat more general way with similar results.

$$
\begin{gathered}
\text { Woolwich Polytechnic, } \\
\text { London, S.E.18. } \\
\text { May 22. }
\end{gathered}
$$

${ }^{1}$ Phil. Mag., 42 (1921).

Ann. Phys., 34 (1911). 